\title{
Prediction of neurodevelopmental outcome in the preterm infant: short latency cortical somatosensory evoked potentials compared with cranial ultrasound
}

\author{
L S de Vries, P Eken, V Pierrat, H Daniels, P Casaer
}

\begin{abstract}
One hundred and twenty six preterm infants, with a gestational age of 34 weeks or less, were studied to compare the predictive value of somatosensory evoked potentials (SEPs) with that of cranial ultrasound. A normal N1 latency was no guarantee of a normal outcome, nor did a persistently delayed $N 1$ latency always correlate with a poor outcome. As a predictor of cerebral palsy, SEPs had a sensitivity of $44 \%$ and a specificity of $92 \%$. The presence of a large haemorrhage (grade IIb/III) or cystic leucomalacia on cranial ultrasound predicted cerebral palsy with a sensitivity of $73 \cdot 6 \%$ and a specificity of $83 \cdot 1 \%$. These results demonstrate that the role of SEPs recorded after median nerve stimulation is limited in preterm infants.
\end{abstract}

(Arch Dis Child 1992;67:1177-81)

With an ever increasing number of surviving low birthweight infants and an increase in the total number of premature infants who develop cerebral palsy, attempts have been made to improve early prediction of neurodevelopmental outcome in these at risk infants. ${ }^{1}$ Cranial ultrasound has played an important part and the presence of extensive cystic periventricular leucomalacia is now acknowledged by many groups to correlate strongly with the development of cerebral palsy. ${ }^{2}{ }^{3}$ It is more complicated to predict the neurodevelopment of infants with other ultrasound abnormalities, like large intraventricular haemorrhages and transient densities, as a large number of these infants survive to be normal in spite of the lesion. ${ }^{245}$ Other modes of investigation, such as magnetic resonance spectroscopy, ${ }^{6}$ electroencephalography, ${ }^{7}$ and somatosensory evoked potentials (SEPs) ${ }^{8-10}$ have been used trying to identify those infants who are to survive with a major handicap. Previous studies using SEPs showed a good correlation between this test and subsequent neurodevelopment, but the number of infants tested in each study tended to be small. ${ }^{8-10}$

The aim of this study was to study prospectively a population of preterm infants in order to evaluate further the role of SEPs in the prediction of neurodevelopmental outcome and to compare the predictive value of SEPs and cranial ultrasound.

\section{Patients and methods}

Over 26 months, 126 premature infants were entered into a prospective correlative study of short latency SEPs and cranial ultrasonography. All infants had a gestational age of 34 weeks or less with a mean gestational age at birth of 30.2 weeks (range 25-34 weeks). Twenty one of the 126 infants were small for gestational age. Informed parental consent was obtained in all infants before entering the study.

ULTRASOUND ASSESSMENT

Ultrasound assessment was performed at least twice a week during the first week of life and twice a week thereafter until discharge, using an ATL UM-4 mechanical sector scanner (5-7.5-10 MHz crystal). Periventricular-intraventricular haemorrhage (PVH-IVH) and periventricular leucomalacia (PVL) were graded as reported previously. ${ }^{11} 12$

\section{PVH-IVH}

Grade I: small germinal layer haemorrhage; grade IIa: germinal layer haemorrhage plus IVH, filling the ventricle $<50 \%$; grade IIb: large IVH distending the ventricle in the acute phase, due to blood filling the ventricle $>50 \%$; grade III: unilateral parenchymal involvement, due to venous or haemorrhagic infarction.

\section{Leucomalacia}

Transient densities: periventricular areas of increased echogenicity, present for seven days or more. Cystic PVL: periventricular areas of increased echogenicity evolving into extensive cystic lesions, involving the occipital with or without the frontoparietal periventricular white matter.

For correlation of cranial ultrasound lesions with subsequent neurodevelopmental outcome the maximum degree of haemorrhage, in case of extension, and the eventual evolution of echogenic areas into cystic lesions or not, were taken into account. 
SEPS

Somatosensory evoked potentials were recorded with a Nihon Kohden Neuropack II or IV. Silver/silver chloride disc electrodes were applied: the negative electrode on $\mathrm{C}^{\prime}$ ' or C4', the positive on $\mathrm{Fz}$ and the neutral one on the lower arm. The impedance was less than $2 \mathrm{kOhms}$. Electrical stimuli of $0.1 \mathrm{msec}$ duration were delivered at a rate of $1 /$ two seconds. A hand held device was used, which was placed on the ventral wrist overlying the median nerve. The stimulation intensity used was that necessary to produce a minimal thumb twitch. Recordings were obtained from the left somatosensory cortex after stimulation of the right median nerve. Recordings were obtained from both hemispheres when a unilateral lesion was present on cranial ultrasound examination. A total of 50-128 responses was averaged through a bandpass of $2-100 \mathrm{~Hz}$. Each trial was repeated two to three times to ensure reproducibility. The analysis time was $200 \mathrm{msec}$. The latency to the peak of the first negative wave (N1) was measured according to the criteria of Desmedt et al. ${ }^{13}$ All infants were tested without sedation after a feed. The filter settings, stimulation rate, and duration were the same as those of Klimach and Cooke so their normal data were used. ${ }^{14}$

All infants were tested for the first time in a stable condition, usually beyond the end of the first week. ${ }^{15}$ Sequential studies were performed in 69 of the 126 infants. In infants with a normal N1 latency on the first examination sequential studies were performed in those who were taking part in a longitudinal study ${ }^{15}$ and in infants who had any clinical deterioration or developed abnormalities on cranial ultrasound. In infants with an N1 latency outside the normal range, that is delayed, or when it was not possible to obtain a response, SEPs were repeated at 40 weeks' postmenstrual age. When the N1 latency had normalised at 40 weeks' postmenstrual age, this last N1 latency was used for correlation with neurodevelopmental outcome.

NEUROLOGICAL ASSESSMENT OF OUTCOME Assessment of outcome was made on clinical examination at discharge from the neonatal unit using the standardised Dubowitz proto$\operatorname{col}^{16}$ and at 6,12 , and 18 months corrected for prematurity using Griffiths's mental development scale and items from the scores of Amiel-Tison and Grenier ${ }^{17}$ and Touwen. ${ }^{18}$ All of the children were last seen when at least 12 months of age corrected for prematurity. Five of the 126 infants were not seen at our follow up clinic and follow up information was obtained from the paediatrician at the referring district general hospital. The children were grouped in three categories. (A) Normal, when the developmental quotient was more than 90 and no neurological, visual, or hearing abnormalities were present. (B) Some abnormality of tone pattern ('dystonia') on at least two separate occasions, but functionally normal. ${ }^{19}$ (C) Definite neurological handicap, cerebral palsy and/or severe hearing or visual defects.

For the purpose of calculating properties such as sensitivity and specificity infants in group B were analysed with those in group A as it is well recognised that infants with transient dystonia only have transient abnormalities in tone. ${ }^{19}$

\section{Results}

SEPS

A total number of 240 recordings were done, with a mean of 1.9 recordings per infant (range 1-11). In 107 of the 126 infants a normal N1 latency was obtained. Fifty eight of these 107 infants had their first examination before the end of the third week, 48 having a normal N1 latency on their first examination and 10 normalising before reaching 40 weeks' postmenstrual age. In 19 infants the N1 latency was delayed, being still outside the $95 \%$ tolerance limit at 40 weeks' postmenstrual age.

Table 1 Neurodevelopmental outcome at $\geq 12$ months and N1 latency at 40 weeks' postmenstrual age

\begin{tabular}{lcclc}
\hline Outcome & Normal & $\begin{array}{l}\text { Transient } \\
\text { dystonia }\end{array}$ & $\begin{array}{l}\text { Cerebral } \\
\text { palsy }\end{array}$ & Total \\
\hline $\begin{array}{c}\text { Normal N1 } \\
\text { latency }\end{array}$ & $82\left(1^{*}\right)$ & 11 & 14 & 107 \\
$\begin{array}{c}\text { Delayed N1 } \\
\text { latency }\end{array}$ & 2 & 6 & $11\left(1^{*}\right)$ & 19 \\
\hline
\end{tabular}

*=Blind due to retinopathy of prematurity.

Table 2 Neurodevelopmental outcome at $\geq 12$ months of age and occurrence and grade of PVH-IVH and leucomalacia

\begin{tabular}{llllc}
\hline Outcome & Normal & $\begin{array}{l}\text { Transient } \\
\text { dystonia }\end{array}$ & $\begin{array}{l}\text { Cerebral } \\
\text { palsy }\end{array}$ & Total \\
\hline Normal scan & 43 & 6 & 0 & 49 \\
PVH-IVH & & & 1 & 20 \\
$\quad$ Grade I-IIa & 15 & 4 & 1 & 10 \\
$\quad$ Grade IIb & 7 & 2 & $7\left(1^{*}\right)$ & 15 \\
$\quad$ Grade III & $8\left(1^{*}\right)$ & 0 & 4 & 20 \\
$\quad \begin{array}{l}\text { Lucomalacia } \\
\quad \text { Transient densities }\end{array}$ & 11 & 5 & 12 & $\underline{12}$ \\
$\quad$ Cystic PVL & 0 & 0 & & 126 \\
\hline
\end{tabular}

*=Blind due to retinopathy of prematurity.

Grade/SEPs

Normal

Delayed N1

1- Па

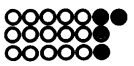

-

Ib

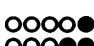

III

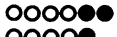

- Normal development

- Cerebral palsy

- Transient dystonia

Figure 1 Correlation of SEPs at 40 weeks' postmenstrual age, ultrasound abnormalities (PVH-IVH) and neurological outcome in 45 infants. 


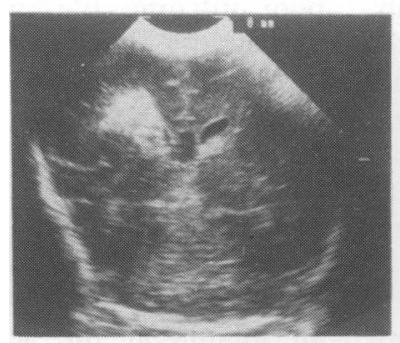

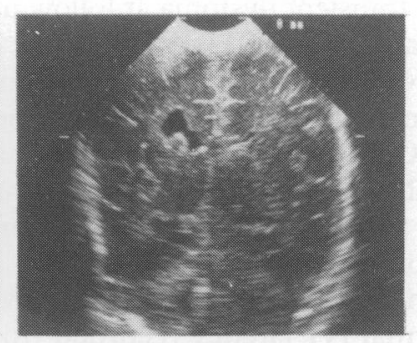

B

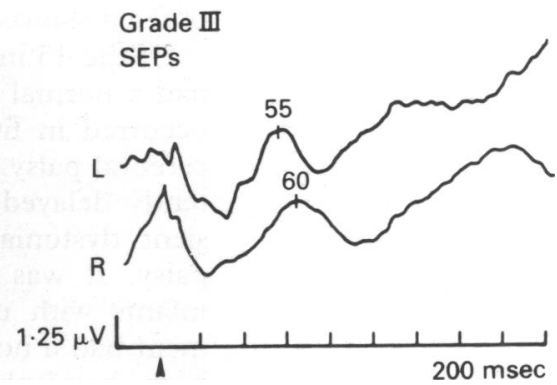

Figure 2 (A) Cranial ultrasound, coronal view, showing a grade III haemorrhage on the right, evolving into a porencephalic cyst. (B) SEPs recorded from the same infant at 36 weeks'postmenstrual age, showing a slight difference in N1 latency, being within the $95 \%$ tolerance limit of Klimach and Cooke in both hemispheres.

Ultrasound/SEPs
Densities
Cystic PVL
\begin{tabular}{|l|l|}
\hline O Normal development \\
- Cerebral palsy \\
\hline
\end{tabular}

Figure 3 Correlation of SEPs at 40 weeks' postmenstrual age, ultrasound abnormalities (leucomalacia) and neurological outcome in 32 infants.

CORRELATION OF SEPS WITH NEUROLOGICAL OUTCOME

A normal N1 latency was not invariably associated with a normal outcome (table 1). Fourteen infants with a normal N1 latency at 40 weeks' postmenstrual age developed cerebral palsy. Longitudinal studies (2-8 times) were performed in 10 of these infants, and six of them initially had a delayed N1 latency.

If the sequential recordings for SEPs were persistently abnormal, the outcome was almost never completely normal. Of the 19 infants with a persistently delayed N1 latency, 11 developed cerebral palsy, six developed transient dystonia, and only two were normal at follow up. Seven of the eight infants with a delayed N1 latency, and who did not develop cerebral palsy, were small for gestational age. As a predictor of cerebral palsy the SEPs had a sensitivity of $44 \%$ and a specificity of $92 \%$.
The predictive value of a positive test was $57 \cdot 8 \%$ and that of a negative test was $87 \%$.

CORRELATION OF CRANIAL ULTRASOUND WITH NEUROLOGICAL OUTCOME

In this cohort a normal ultrasound scan was never associated with the development of cerebral palsy and only six of the 49 infants with a normal scan developed transient dystonia (table 2). Of the 25 infants with large intraventricular haemorrhage with (III) or without (IIb) parenchymal involvement, 15 were found to be normal at early follow up and eight developed cerebral palsy. Of the 20 infants with transient densities, only 11 were normal at follow up, six developed transient dystonia, and four developed cerebral palsy. Cystic PVL was invariably associated with a poor outcome and all 12 infants developed cerebral palsy.

As a predictor for cerebral palsy severe ultrasound abnormalities (grade IIb-III haemorrhage and cystic PVL) had a sensitivity of $73.6 \%$, a specificity of $83.1 \%$, a positive predictive value of $45 \cdot 1 \%$, and a negative predictive value of $94 \cdot 3 \%$.

\section{CORRELATION OF SEPS AND ULTRASOUND} FINDINGS WITH NEURODEVELOPMENTAL OUTCOME.

All 35 infants who were of appropriate weight for gestational age without ultrasound abnormalities had a normal N1 latency and only one of them developed transient dystonia. Of the 14 infants who were small for gestational age without ultrasound abnormalities, five infants
A

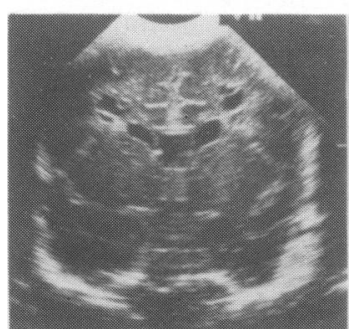

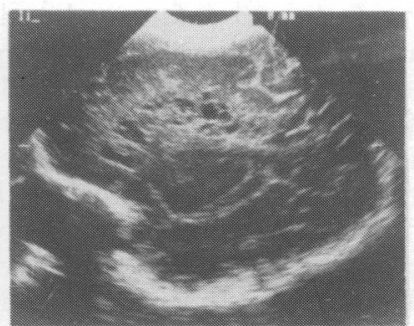

Cystic PVL SEPS

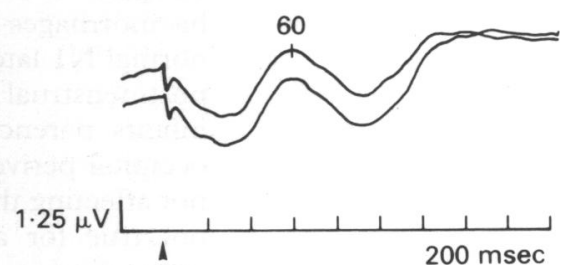

Figure 4 (A) Cranial ultrasound scan, showing extensive cystic PVL in the coronal (left) and parasagittal (right) view. (B) SEPs recorded from the same infant at 35 weeks' postmenstrual age, showing an N1 latency at 60 msec, which is within the $95 \%$ tolerance limit of Klimach and Cooke. 
had a persistently delayed N1 latency and three of them showed transient dystonia at follow up.

Of the 45 infants with PVH-IVH (fig 1), 40 had a normal N1 latency. Transient dystonia occurred in five of them and five developed cerebral palsy. Of the five infants with persistently delayed N1 latency one showed transient dystonia and four developed cerebral palsy. It was of interest that 11 of the 15 infants with unilateral parenchymal involvement had a normal N1 latency recorded from both hemispheres (fig 2). Three of them developed cerebral palsy, one a hemiplegia and the other two, with a porencephalic cyst in the occipital white matter, suffered from hypotonic cerebral palsy and visual impairment. Four of the 15 infants had a persistently delayed N1 latency, being delayed on both sides in all four. All four developed a hemiplegia with upper motor neuron signs present on both sides.

Seventeen of the 20 infants with transient densities had a normal N1 latency (fig 3). Transient dystonia occurred in three and three developed cerebral palsy. Of the three infants with a delayed N1 latency, two developed transient dystonia and one cerebral palsy. Of the 12 infants with cystic PVL, six had a normal N1 latency, but all infants developed cerebral palsy (fig 4).

Only seven of the 77 infants with ultrasound abnormalities were small for gestational age. Three of them had a normal N1 latency with subsequently a normal outcome. Four infants had a persistently delayed N1 latency associated with a grade III haemorrhage in one, who developed cerebral palsy, and associated with transient densities in the other three, one of whom developed cerebral palsy and the other two developed transient dystonia.

When the presence of major ultrasound abnormalities (grade IIb-III, cystic PVL) and a delayed N1 latency were taken together as a predictor for cerebral palsy, the sensitivity was $40 \%$, specificity $100 \%$, positive predictive value $100 \%$, and negative predictive value $78 \cdot 4 \%$.

\section{Discussion}

Previous studies, using SEPs in premature infants, for prediction of subsequent neurodevelopmental outcome have been quite promising. ${ }^{8-10}$ The predictive value in the present study, looking at a large cohort of infants with and without abnormalities on cranial ultrasound, was not as good as expected. A normal N1 latency was no guarantee of a normal outcome. Fourteen of the 25 infants who developed cerebral palsy, both related to large haemorrhages as well as to cystic PVL, had a normal N1 latency at or even before 40 weeks' postmenstrual age. Although in some of these infants parenchymal involvement was in the occipital periventricular white matter, possibly not affecting the somatosensory tracts, this was not true for all cases. In some infants with extensive cystic leucomalacia, cysts were noted in the frontoparietal and occipital periventricular white matter. A persistently delayed N1 latency also did not always correlate with a poor outcome. Eleven of the 19 infants with a persistently delayed N1 latency developed cerebral palsy. Seven of the eight infants with a delayed N1 latency, who did not develop cerebral palsy, were small for gestational age and five of these seven infants did not have any abnormalities on cranial ultrasound.

Due to the high turnover in our tertiary intensive care unit it was not possible to study all infants at the same postmenstrual age. However, all infants with a delayed N1 latency on the initial recording had repeat studies until normalisation occurred or the infant had reached 40 weeks' postmenstrual age. Of the 14 infants who developed cerebral palsy in spite of a normal N1 latency at $\mathbf{4 0}$ weeks' postmenstrual age, 10 had longitudinal studies showing a change from a delayed N1 latency to a normal N1 latency in six infants and a repeatedly normal $\mathrm{N} 1$ latency in the other four infants. A delayed N1 latency after an initially normal N1 latency was noted only twice among the 69 infants studied sequentially. This was in both cases associated with posthaemorrhagic ventricular dilatation, being reversible in one, after shunt insertion. ${ }^{20}$

Only 35 infants admitted during the study period were of appropriate weight for gestational age and had no abnormalities on cranial ultrasound. Calculating the regression equation of the N1 latency for these 35 infants showed this equation to be almost equal to that of Klimach and Cooke. ${ }^{14}$ As the same filter settings and stimulation rate were used, and their normal data were collected from 102 infants, it was decided to use their normal data.

The findings in the present cohort differ from previous reports in the literature. Klimach and Cooke studied 30 low birthweight infants with abnormalities on cranial ultrasound. ${ }^{8}$ All infants with a normal N1 latency were normal at follow up and six infants with a persistently delayed N1 latency had an abnormal outcome. Majnemer et al presented preliminary findings of 44 low birthweight infants. ${ }^{9}$ Infants with absent or persistently delayed responses were found to have a major handicap at follow up. The SEPs had a sensitivity of $79 \%$ and a specificity of $88 \%$. Laget et al studied 43 infants with a hemiplegia, between 1 month and 13 years of age, and found absent responses in 12 and a decrease in amplitude in the other 31 infants from the contralateral hemisphere. ${ }^{21}$ Of the six infants described in this present study who developed a hemiplegia, five had a delayed N1 latency and this was present in both hemispheres in four of them. This is in agreement with clinical findings as most of the infants who developed a hemiplegia after a grade III haemorrhage also tend to have upper motor neuron signs on the other side of the body. Using positron emission tomography in infants with this type of lesion Volpe et al showed that preterm infants with a grade III haemorrhage had changes in cerebral blood flow in the non-affected hemisphere as well. ${ }^{22}$ Willis et al studied 39 infants with PVH-IVH at 2,4 , and 6 months' corrected age. ${ }^{10}$ Their 
findings are more similar to ours as they found that $36 \%$ of the infants with normal SEPs at 2 months' corrected age did go on to develop moderate or severe abnormalities. Only $14 \%$ of the infants with normal SEPs on three occasions had a poor outcome. As the aim of our study was to assess whether it was possible to improve early prediction, the infants were studied only until the age of $\mathbf{4 0}$ weeks' postmenstrual age.

In none of these previous studies was a distinction made between infants who were of appropriate weight or small for gestational age. Gallai et al, however, also drew attention to a delay (of $5 \mathrm{msec}$ ) in $\mathrm{N} 1$ latency in small for gestational age infants. ${ }^{23}$ Our findings suggest that especially when there is an increase in N1 latency it should be taken into account whether the infant is small for gestational age or not.

When a comparison was made between SEPs and cranial ultrasound, the latter technique was noted to be more sensitive. All infants with an ultrasound diagnosis of cystic PVL went on to develop cerebral palsy, but only six of the 12 infants had a delayed N1 latency. When the presence of major ultrasound abnormalities (grade IIb-III, cystic PVL) and a delayed N1 latency were taken together as a predictor for cerebral palsy, the sensitivity was not better, but specificity and positive predictive value both became a $100 \%$. In an earlier study, using SEPs in full term infants with postasphyxial encephalopathy, SEPs of the median nerve appeared to be of value when trying to predict subsequent neurodevelopmental outcome, ${ }^{24}$ and this was recently confirmed by other groups. ${ }^{25}{ }^{26} \mathrm{It}$ is possible that SEPs after stimulation of the median nerve are less useful in the preterm infant as the legs are usually more severely affected than the arms. At present a study is being undertaken in our unit to compare SEPs of the posterior tibial nerve and the median nerve to find out whether the SEPs of the posterior tibial nerve will be of better predictive value.

SEPs of the median nerve appear to be of limited value as an early predictor of subsequent neurodevelopmental outcome in the preterm infant and should probably not be performed routinely in all low birthweight infants.

Dr P Eken was supported by a grant from the Praeventiefonds, The Hague, The Netherlands and Dr V Pierrat by a gran from COMETT, Brussels, Belgium.

1 Hagberg B, Hagberg G, Zetterstrom R. Decreasing perinatal mortality-increase in cerebral palsy morbidity? Acto Paediatr Scand 1989;78:664-70.
2 De Vries LS, Dubowitz LMS, Dubowitz V, et al. Predictive value of cranial ultrasound: a reappraisal. Lancet 1985 ii: $137-40$.

3 Fawer CL, Diebold P, Calame A. Periventricular leukomalacia and neurodevelopmental outcome in preterm infants. Arch Dis Child 1987;62:30-6.

4 Appleton RE, Lee REJ, Hey EN. Neurodevelopmental outcome of transient neonatal intracerebral echodensities. Arch Dis Child 1990;65:27-9.

5 De Vries LS, Regev R, Pennock JM, Wigglesworth JS, Dubowitz LMS. Ultrasound evolution and later outcome of infants with periventricular densities. Early Hum Dev 1988;16:225-33.

6 Hamilton PA, Hope PL, Cady EB, Delpy DT, Wyatt JS, Reynolds EOR. Impaired energy metabolism in the brains of newborn infants with increased cerebral echodensities. Lancet 1986;i:1242-6.

7 Connell JA, De Vries LS, Oozeer RC, Regev R, Dubowitz LMS, Dubowitz V. Predictive value of early continuous EEG monitoring in ventilated preterm infants with IVH. Pediatrics 1988;82:337-43.

8 Klimach VJ, Cooke RWI. Short latency somatosensory cortical evoked responses of preterm infants with ultrasound abnormalities. Dev Med Child Neurol 1988; 30:215-21.

9 Majnemer A, Rosenblatt B, Riley PS. Prognostic significance of multimodality evoked response abnormalities in high risk low birth weight infants. Pediatr Neurol 1990; 6:367-74.

10 Willis J, Duncan MC, Bell R, Pappas F, Moniz M Somatosensory evoked potentials predict neuromotor outcome after periventricular haemorrhage. Dev Med outcome after periventricular
Child Neurol 1989;31:435-9.

11 Levene MI, Fawer C-L, Lamont RF. Risk factors in the development of intraventricular haemorrhage in the preterm neonate Arch Dis Child 1982;57:410-7.

12 De Vries LS, Eken P, Dubowitz LMS. The spectrum of leukomalacia using cranial ultrasound. Behav Brain Res 1992;49:1-6.

13 Desmedt JE, Brunko E, Debecker J. Maturation of the somatosensory evoked potentials in normal infants and children with special reference to the early N1 component. Electroencephalogr Clin Neurophysiol 1974;40 ponent.

14 Klimach V, Cooke RWI. Maturation of the neonatal somatosensory evoked responses in preterm infants. Dev Med Child Neurol 1988;30:208-14

15 Pierrat V, De Vries LS, Minami T, Casaer P. Somatosensory evoked potentials and adaptation to extrauterine life: a longitudinal study. Brain Dev 1990; 12:376-80.

16 Dubowitz LMS, Dubowitz V. The neurological assessment of the preterm and full-term infant. Clinics of developmental medicine. No 79. London: SIMP/Heinemann, 1981

17 Amiel-Tison C, Grenier A. Evaluation neurologique du nouveau-né et du nourisson. Paris: Masson, 1980.

18 Touwen BLC. Examination of the child with minor neurological dysfunction. Clinics in developmental medicine. No 71. London: SIMP/Heinemann, 1979.

19 Drillien CM. Abnormal neurologic signs in the first year of life in low-birth weight infants: possible prognostic significance. Dev Med Child Neurol 1972;14:575-84

20 De Vries LS, Pierrat V, Minami T, Casaer P. Short latency cortical somatosensory evoked potentials in infants with cortical somatosensory evoked potentials in in

21 Laget P, Salbreux R, Raimbault J, d'Allest AM, Mariani J. Relationship between changes in somesthetic evoked responses and electroencephalographic findings in the child with hemiplegia. Dev Med Child Neurol 1976;18 620-31.

22 Volpe JJ, Perlman JM, Hill A, McMenamin J. Cerebral blood flow velocity in the human newborn: the value of its determination. Pediatrics 1982;70:147-52.

23 Gallai V, Mazzotta G, Cagini L, del Gatto F, Angelotti F Maturation of SEPs in preterm and full-term neonates. In: Gallai V, ed. Maturation of the CNS and evoked potentials. Amsterdam: Excerpta Medica, 1986: 95-106.

24 De Vries LS, Pierrat V, Eken P, Minami T, Daniels H, Casaer P. Prognostic value of somatosensory evoked potentials for adverse outcome in full-term infants with potentials for adverse outcome in full-term
birth asphyxia. Brain Dev 1991;13:320-5.

25 Gibson NA, Graham M, Levene MI. Somatosensory evoked potentials and outcome in perinatal asphyxia. evoked potentials and outcom
Arch Child 1992;67:393-8.

26 Taylor MJ, Murphy WJ, Whyte HE. Prognostic reliability of SEPs and VEPs in asphyxiated term infants. Dev Med Child Neurol 1992;34:507-15. 